\title{
Reconstructing Popov v. Hayashi in a framework for argumentation with structured arguments and Dungean semantics
}

\author{
Henry Prakken
}

Published online: 11 March 2012

(C) The Author(s) 2012. This article is published with open access at Springerlink.com

\begin{abstract}
In this article the argumentation structure of the court's decision in the Popov v. Hayashi case is formalised in Prakken's (Argument Comput 1:93-124; 2010) abstract framework for argument-based inference with structured arguments. In this framework, arguments are inference trees formed by applying two kinds of inference rules, strict and defeasible rules. Arguments can be attacked in three ways: attacking a premise, attacking a conclusion and attacking an inference. To resolve such conflicts, preferences may be used, which leads to three corresponding kinds of defeat, after which Dung's (Artif Intell 77:321-357; 1995) abstract acceptability semantics can be used to evaluate the arguments. In the present paper the abstract framework is instantiated with strict inference rules corresponding to first-order logic and with defeasible inference rules for defeasible modus ponens and various argument schemes. The main techniques used in the formal reconstruction of the case are rule-exception structures and arguments about rule validity. Arguments about socio-legal values and the use of precedent cases are reduced to arguments about rule validity. The tree structure of arguments, with explicit subargument relations between arguments, is used to capture the dependency relations between the elements of the court's decision.
\end{abstract}

Keywords Legal argument · Argumentation structure . Logics for argumentation

H. Prakken $(\bowtie)$

Department of Information and Computing Sciences, Utrecht University, Utrecht, The Netherlands e-mail: henry@cs.uu.nl

H. Prakken

Faculty of Law, University of Groningen, Groningen, The Netherlands 


\section{Introduction}

This article presents a formalisation of the Popov v. Hayashi case in an instance of Prakken's (2010) framework for argument-based inference with structured arguments. This framework further develops Amgoud et al. (2006)'s attempt to integrate work on rule-based argumentation of Pollock (1994), Vreeswijk (1997), Prakken and Sartor (1997) and others. It defines arguments as inference trees formed by applying two kinds of inference rules, strict and defeasible rules. This naturally leads to three ways of attacking an argument: attacking a premise, attacking a conclusion and attacking an inference. To resolve such conflicts, preferences may be used, which leads to three corresponding kinds of defeat: undermining, rebutting and undercutting defeat. The framework is abstract in that it applies to any set of inference rules, as long as it is divided into strict and defeasible ones, and to any logical language with a contrary relation defined over it. Moreover, since it associates each knowledge base with a set of arguments ordered by a binary relation of defeat, the acceptability status of arguments can be defined in terms of Dung's (1995) well-understood abstract approach to argument acceptability.

The choice for tree-structured arguments based on two types of inference rules arguably is very natural, not only in light of logic and argumentation theory but also when looking at argumentation as it occurs in human thinking and dialogue. The notion of arguments as trees of inferences is very common in standard logic and in argumentation theory, and it is the basis of many software tools for argument visualisation. Moreover, in actual argumentation humans often express their arguments as claims supported with one or more premises, which can in turn be supported with further premises, and so on, which results in tree structures. The tree form is therefore very suitable for capturing the dependency relations between the various elements of the court's decision. Finally, the framework is very suited for modelling reasoning with argumentation schemes, by regarding such schemes as defeasible inference rules.

The instance of the framework assumed in this paper will draw on my previous work (partly with others) on rule-based argumentation (Prakken and Sartor 1997), reasoning with argument schemes (Bex et al. 2003; Prakken 2005a), accrual of arguments (Prakken 2005b) and reasoning under burden of proof (Prakken and Sartor 2009).

The main purpose of the present reconstruction is theoretical, namely, to test the adequacy of the framework of Prakken (2010) for giving a formal reconstruction of the Popov v. Hayashi case. More precisely, the object of the formalisation is the relevant facts and rules stated by judge McCarthy and the arguments constructed by him on the basis of these facts and rules. The reconstruction will not be descriptive but rational: the aim is to reconstruct the judge's reasoning as being, as far as possible, rationally sound. Assuming that the judge's reasoning in the case is by and large sound, the extent to which such a reconstruction is possible indicates the suitability of the formal framework.

A secondary aim of the case study is more practical, namely, to give a reconstruction as could be made by lawyers or law students with so-called sensemaking software. With such software, e.g. Reed and Rowe's (2004) 
Araucaria or Van den Braak's (2010) Avers, users can draft their own arguments or analyse given arguments, in order to improve the quality of their arguments or to better grasp the issues in a case. The present case study is also meant to test the suitability of the framework of Prakken (2010) as a theoretical basis for the design of sensemaking software for legal applications. Since users of such software will not be experts in knowledge representation, the main focus of this case study is on the general structure of arguments and their relations instead of on detailed knowledge representation issues, such as time, action, causation and intention. The only representation issues that will be discussed in detail are the representation of the validity of rules and of rule-exception structures, since these issues turn out to be crucial for capturing judge McCarthy's reasoning. The focus on sensemaking applications also means that the reconstruction of the case is ideally as close to the judge's actual formulation as possible, within the bounds defined by the primary aim to give a rational instead of purely descriptive reconstruction of the case.

This article is organised as follows. First in Sect. 2 the instance of Prakken's (2010) framework used in this article will be semi-formally presented. Then in Sect. 3 the formalisation of the case will be described in two parts: the facts (Sect. 3.1) and the legal analysis (Sect. 3.2). This section only presents the facts and rules discussed by judge McCarthy; the arguments that he constructs from them are presented in Sect. 4. The formalisation will be evaluated in Sect. 5.

\section{The formalism}

In this section the instance of Prakken's (2010) framework used in this article will be semi-formally presented. For the full details the reader is referred to the technical report.

\subsection{The basic definitions}

The basic notion of the present framework is that of an argumentation system, which extends the familiar notion of a proof system with a distinction between strict and defeasible inference rules and a preference ordering on the defeasible inference rules. More precisely:

An argumentation system is a tuple $A S=(\mathcal{L},-, \mathcal{R}, \leq)$ where

- $\mathcal{L}$ is a logical language,

- $\quad$ - is a contrariness function from $\mathcal{L}$ to $2^{\mathcal{L}}$,

- $\mathcal{R}=\mathcal{R}_{s} \cup \mathcal{R}_{d}$ is a set of strict $\left(\mathcal{R}_{s}\right)$ and defeasible $\left(\mathcal{R}_{d}\right)$ inference rules such that $\mathcal{R}_{s} \cap \mathcal{R}_{d}=\emptyset$

- $\leq$ is a partial preorder on $\mathcal{R}_{d}$.

In this article the logical language $\mathcal{L}$ will be that of first-order logic augmented with a defeasible conditional $\leadsto$. To avoid excessive formalism, the facts and rules will be expressed in a semi-formal way. The contrariness function specifies which 
formulas cannot be accepted together. In the present paper it will be used to capture classical negation (formulas $\varphi$ and $\neg \varphi$ are each other's contraries) and to express some domain-specific conflict relations (e.g. ' $x$ must return the baseball to $y$ ' and ' $x$ and $y$ must equally share the value of the baseball' will be each other's contraries).

Strict rules are of the form $\varphi_{1}, \ldots, \varphi_{n} \rightarrow \varphi$, interpreted as 'if the antecedents $\varphi_{1}, \ldots, \varphi_{n}$ are acceptable, then the consequent $\varphi$ must be accepted, no matter what'. Defeasible rules are written as $\varphi_{1}, \ldots, \varphi_{n} \Rightarrow \varphi$, meaning 'if the antecedents are acceptable, then the consequent must be accepted if there is no good reason not to accept it'. Although the general framework allows for domain-specific inference rules, as used by e.g. Prakken and Sartor (1997), the present instance will only use inference rules that express general inference patterns; domain-specific rules will instead be represented in $\mathcal{L}$ with the material implication $\supset$ (if exceptionless) or with the connective $\leadsto$ (if subject to exceptions). The set $\mathcal{R}_{s}$ of strict inference rules will be assumed to contain all deductively valid propositional and first-order inferences, while the set $\mathcal{R}_{d}$ of defeasible inference rules contains a defeasible modus ponens rule for $\leadsto$ and some argument schemes specified below in Sect. 2.2. The preference ordering $\leq$ will not be needed and is therefore not specified.

Arguments are constructed from a knowledge base $\mathcal{K}$ which, inspired by Gordon et al. (2007), is assumed to contain four kinds of formulas:

- $\mathcal{K}_{n}$ is a set of (necessary) axioms. Intuitively, arguments cannot be attacked on their axiom premises.

- $\mathcal{K}_{p}$ is a set of ordinary premises. Intuitively, arguments can be attacked on their ordinary premises, and whether this results in defeat must be determined by comparing the attacker and the attacked premise.

- $\mathcal{K}_{a}$ is a set of assumptions. Intuitively, arguments can be attacked on their assumptions, where these attacks always succeed.

- $\mathcal{K}_{i}$ is a set of issues. Intuitively, arguments of which the premises include an issue are never acceptable: an issue must always be backed with a further argument.

In the present paper only ordinary premises and assumptions will be needed. In the general framework a partial priority ordering is assumed on $\mathcal{K} \backslash K_{n}$. Since it will not be needed for the present formalisation, it is not specified here.

Arguments can be built by chaining inference rules into trees, starting with elements from the knowledge base. The following definition is adapted from Vreeswijk (1997). In this definition, for any argument $A$, the function Prem returns all the formulas of $\mathcal{K}$ (called premises) used to build $A$, Conc returns $A$ 's conclusion and Sub returns all of $A$ 's sub-arguments.

An argument $A$ on the basis of a knowledge base $\left(\mathcal{K}, \leq^{\prime}\right)$ in an argumentation system $\left(\mathcal{L},{ }^{-}, \mathcal{R}, \leq\right)$ is:

1. $\varphi$ if $\varphi \in \mathcal{K}$ with: $\operatorname{Prem}(\mathrm{A})=\{\varphi\} ; \operatorname{Conc}(\mathrm{A})=\varphi ; \operatorname{Sub}(\mathrm{A})=\{\varphi\}$.

2. $A_{1}, \ldots A_{n} \rightarrow / \Rightarrow \psi$ if $A_{1}, \ldots, A_{n}$ are arguments such that there exists a strict/ defeasible rule $\operatorname{Conc}\left(\mathrm{A}_{1}\right), \ldots, \operatorname{Conc}\left(\mathrm{A}_{\mathrm{n}}\right) \rightarrow / \Rightarrow \psi$ in $\mathcal{R}_{s} / \mathcal{R}_{d}$. 


$$
\begin{aligned}
\operatorname{Prem}(A & =\operatorname{Prem}\left(A_{1}\right) \cup \ldots \cup \operatorname{Prem}\left(A_{n}\right), \\
\operatorname{Conc}(A) & =\psi \\
\operatorname{Sub}(A) & =\operatorname{Sub}\left(A_{1}\right) \cup \ldots \cup \operatorname{Sub}\left(A_{n}\right) \cup\{A\} .
\end{aligned}
$$

Consider by way of example a knowledge base in an argumentation system with

$$
\begin{aligned}
& \mathcal{R}_{s}=\{p, q \rightarrow s ; u, v \rightarrow w\} ; \mathcal{R}_{d}=\{p \Rightarrow t ; s, r, t \Rightarrow v\} \\
& \mathcal{K}_{n}=\{q\} ; \mathcal{K}_{p}=\{p, u\} ; \mathcal{K}_{a}=\{r\}
\end{aligned}
$$

An argument for $w$ is displayed in Fig. 1. The type of a premise is indicated with a superscript.

Formally the argument and its subarguments are written as follows:

$$
\begin{array}{ll}
A_{1}: p & A_{5}: A_{1} \Rightarrow t \\
A_{2}: q & A_{6}: A_{1}, A_{2} \rightarrow s \\
A_{3}: r & A_{7}: A_{5}, A_{3}, A_{6} \Rightarrow v \\
A_{4}: u & A_{8}: A_{7}, A_{4} \rightarrow w
\end{array}
$$

We have that

$$
\begin{aligned}
\operatorname{Prem}\left(\mathrm{A}_{8}\right) & =\{p, q, r, u\} \\
\operatorname{Conc}\left(\mathrm{A}_{8}\right) & =w \\
\operatorname{Sub}\left(\mathrm{A}_{8}\right) & =\left\{A_{1}, A_{2}, A_{3}, A_{4}, A_{5}, A_{6}, A_{7}, A_{8}\right\}
\end{aligned}
$$

Arguments can be attacked on their premises (except on axioms) and on their applications of defeasible inference rules. The latter can be done in two ways: by rebutting the argument, i.e, by providing an argument for a contrary conclusion, or by undercutting the argument, i.e, by concluding that the attacked inference rule does not apply to the case at hand. Rebutting and undercutting attacks can be directed towards an argument's final conclusion or towards one of its intermediate conclusions, that is, to a conclusion of a subargument. In our example, argument $A_{8}$

Fig. 1 An argument

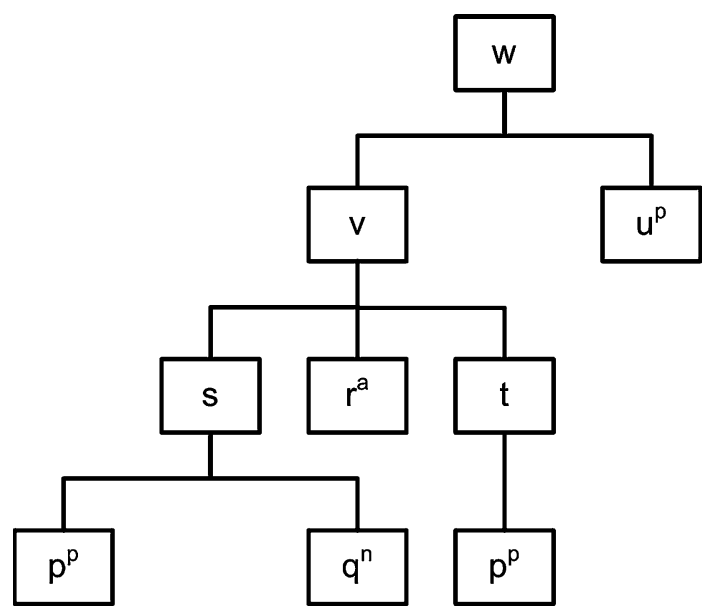


can be attacked on its premises $p$ and $r$ but not on $q$. It can be rebutted on $s$ and $w$ and undercut on the inferences of $t$ and $v$. In general, premise attacks and rebuttals must be resolved with the priority orderings on $\mathcal{R}_{d}$ and $\mathcal{K}$ to see whether the attack results in defeat. However, since in the present reconstruction these orderings are not needed, all three forms of attack will result in defeat.

Having thus defined a set of arguments ordered by a binary defeat relation, any of Dung's (1995) semantics can be used to define the acceptability status of arguments. It will turn out that for present purposes their differences do not matter and their application is straightforward. For this reason these semantics will not be discussed here.

\subsection{The argument schemes}

Because of its distinction between strict and defeasible inference rules, the framework is suitable for modelling reasoning with argument schemes, which currently is an important topic in the computational study of (legal) argument (Walton et al. 2008; Gordon et al. 2007; Atkinson and Bench-Capon 2007). Argument schemes are stereotypical non-deductive patterns of reasoning, consisting of a set of premises and a conclusion that is presumed to follow from them. Uses of argument schemes are evaluated in terms of critical questions specific to the scheme. A natural way to formalise reasoning with argument schemes is to regard them as defeasible inference rules and to regard critical questions as pointers to counterarguments; cf. (Verheij 2003; Bex et al. 2003). Since attacks on premises and conclusions are captured by the general definitions of rebutting and premise attack, below only undercutters of argument schemes will be listed.

First two schemes needed for reasoning with and about rules will be defined. As said above, domain-specific defeasible rules will be represented in the object language $\mathcal{L}$ with a connective $\rightsquigarrow$. (Such domain-specific defeasible rules must not be confused with the defeasible inference rules from $R_{d}$, which are specified in the metalanguage of $\mathcal{L}$. Below the context will disambiguate the use of the term 'rule'). Since in the reconstruction of the case the validity of (domain-specific) rules will turn out to be crucial, Hage's (1996) conceptual distinction (though not his formalisation) between the general validity of rules and their applicability to a case at hand will be adopted. To capture this distinction, an argument scheme will be used that is inspired by recent work of Sartor (2008) and Bex (2009). (The following schemes assume that the rule in $\mathcal{L}$ of the form $\varphi \leadsto \psi$ is named by term $\mathcal{L}$-term $r$.)

\section{Rule validity scheme:}

$A S_{r v}: \quad$ rule $r$ is valid $\rightarrow \varphi \rightsquigarrow \psi$

(Since this is a strict rule, it has no undercutters.) The Popov v. Hayashi case contains several explicit arguments about rule validity but most rules are simply stated; in those cases their validity will below be expressed as an assumption-type premise. It will turn out that some rules of the case are legal while others are empirical commonsense rules. Both types of rules will be represented with $\leadsto$; their different nature can be captured with different validity conditions. 
Once a rule has been derived with the rule validity scheme, it can be applied with the following defeasible modus ponens argument scheme. This scheme has one undercutter $(U)$, namely that the rule is not applicable, which can be used to express exceptions to the rule.

\section{Defeasible modus ponens:}

$$
\begin{array}{ll}
A S_{d m p}: & \varphi \rightsquigarrow \psi, \varphi \Rightarrow \psi \\
U: & \text { rule } r \text { is not applicable }
\end{array}
$$

Note that while thus all rules are applicable by default (since inapplicability means that there is an exception to the rule), their validity can only be assumed when warranted by a particular assumption-type premise for a particular rule. It seems too strong in general to assume that rules are valid by default.

Later in Sect. 3.2 the need for an analogical rule application scheme will become apparent.

Next some argument schemes are needed to reconstruct the arguments about the facts, namely, the witness testimony scheme familiar from the literature on argument schemes, plus a scheme for arguments from videotape taken from Wyner et al. (2007). The latter scheme will be used to model judge McCarthy's factual inferences from the video recording that was made of the incident. The witness testimony scheme has a general undercutter in case the witness is not credible. Further rules can describe cases in which witnesses are not credible; these further rules then correspond to the familiar lists of critical questions for this scheme (both schemes below assume a naming convention for elements of $\mathcal{L}$ ).

Witness testimony scheme:

$A S_{w t}: \quad$ Witness $w$ says that $\varphi \Rightarrow \varphi$

$U$ : $\quad$ Witness $w$ is not credible

The argument scheme from video tape has a similar form.

Video tape scheme:

$A S_{v t}: \quad$ Video recording $v$ shows that $\varphi \Rightarrow \varphi$

$U$ : $\quad$ Video recording $v$ is not reliable

Finally, Prakken's (2005b) scheme for accrual of arguments (acc) will be used. If there are multiple defeasible arguments for the same conclusion, this scheme combines them in a new accrual argument, for which a new strength can be determined. Since the use of this scheme is not crucial for the case, the reader is referred to Prakken (2005b) for the formal details.

\section{The formalisation}

This section presents the reconstruction of the case. The reconstruction will not be purely descriptive but to some extent 'rational' in that incomplete lines of reasoning 
will be completed in a way that makes them fit the theoretical model. Since this theoretical model is intended to formulate principles for rational argumentation, these completions will therefore make the incomplete arguments 'valid' in the sense defined by the theoretical model. However, completions must still be close to the actual text of the decision; speculations as to what the judge could have had in mind will be avoided as much as possible.

\subsection{The facts}

In the formula names below $e$ is used for evidence, $f$ for material facts and $l$ for legal (or ultimate) facts. Furthermore, rules are named with $r$ or, if proposed by legal experts or the judge, with their initials.

Judge McCarthy observes that the following facts are all proven by the video tape and supporting witness statements. McCarthy explains in some detail that the video tape is reliable evidence since it was was shot from a position with clear view on the events. In two cases he makes statements on the reliability of witnesses, namely, if he wants to reject their testimony. One of these cases is formalised below in Fig. 3. Since apart from these (still minor) issues the proof of the facts was not an issue in the case, their derivation from the evidence with the witness and video tape schemes will be left implicit. The main facts concerning what happened during the baseball match are then as follows.

$f_{1}$ : Popov stopped the forward motion of the baseball

$f_{2}$ : the baseball was in the glove of Popov

$f_{3}$ : Popov was in motion

$f_{4}$ : an out of control mob of unknown persons illegally attacked Popov

$f_{5}$ : Hayashi did not attack Popov

$f_{6}$ : Popov lost contact with the baseball during the violent attack

$f_{7}$ : Hayashi retrieved the baseball

$f_{8}$ : Popov intended to control the baseball

$f_{9}$ : the baseball was hit into the stands

As a matter of fact, McCarthy briefly discusses the proof of $f_{4}$. After observing that several witnesses contradicted this fact, he says that the videotape clearly establishes its truth and (footnote 4) that these witnesses made no true statements "in other particulars", because of which their entire testimony must be rejected. His arguments can be easily reconstructed with the schemes from witness testimony and video tape but since the truth of $f_{4}$ was just a minor issue in the case, they will for reasons of space not be formalised.

Later it will turn out that further material facts are needed; they will not be listed in the text but indicated in the argument graphs of Sect. 4. The same holds for procedural and legal facts unless listed below.

\subsection{Legal analysis}

A central issue in the case is whether certain rules should be adopted. This will be modelled in terms of rule validity. In the case three sources of rule validity are relevant. 
$v_{1}$ : rule $r$ was used in precedent case $c \leadsto$ rule $r$ is valid

$v_{2}$ : rule $r$ is proposed by legal expert $e \leadsto$ rule $r$ is valid

$v_{3}$ : rule $r$ is adopted by relevant practice $p \leadsto$ rule $r$ is valid

(The reason why these rules are not modelled as argument schemes is that they are not general patterns of reasoning but specific rules about the validity of legal rules and as such themselves debatable.) This list is not meant to be exhaustive in general but (with a further rule to be specified below) suffices for the present case. An interesting philosophical question is how the validity of these rules can be supported and whether this leads to an infinite regress. However, for present purposes their validity can simply be assumed as as assumption-type premises.

The main legal issue is whether Popov achieved possession or the right to possession as he attempted to catch and hold on to the ball (p. 5). If Popov (who has the burden of persuasion), succeeds in proving this, then further rules justify liability of Hayashi if he does not return the baseball on demand of Popov. Leaving the precise nature of these further rules implicit, this can be formalised as follows:

$r_{1}$ : ( $x$ has possession of $y \vee x$ has a right to possession of $y$ ) $\wedge z$ holds $y \wedge x$ demands $z$ to return $y \leadsto z$ must return $y$ to $x$

$r_{1}: x$ does not have possession of $y \wedge x$ has no right to possession of $y \wedge z$ holds $y \wedge x$ demands $z$ to return $y \leadsto z$ does not have to return $y$ to $x$

(Since both formulas are based on the same rule, they are given the same name.) According to footnote 14, this rule is apparently based on a precedent:

\section{$l_{1}: \quad r_{1}$ was used in Metropolitan Life Insurance Company v. San Francisco Bank}

With this fact, the validity of $r_{1}$ can be derived with validity rule $v_{1}$.

\subsubsection{Possession}

McCarthy first discusses whether Popov has achieved possession of the ball (the first disjunct of the first condition of $r_{1}$ ). The following thee rules state that spectators of baseball games who catch or retrieve a baseball gain possession of the ball.

$r_{2}$ : a baseball is hit into the stands $\leadsto$ the baseball becomes abandoned property

$r_{3}$ : a baseball is abandoned property $\wedge x$ catches the baseball $\leadsto x$ has possession of the baseball

$r_{4}$ : a baseball is abandoned property $\wedge x$ retrieves the baseball $\leadsto x$ has possession of the baseball

Apparently, these rules are generally accepted by baseball fans. Then combined with an obvious implicit commonsense rule, their validity can be derived from rule $v_{3}$.

$l_{2}$ : $\quad$ rule $r_{2}$ confirms to the long-standing expectations of baseball fans

$l_{3}$ : rule $r_{3}$ confirms to the long-standing expectations of baseball fans

$l_{4}$ : $\quad$ rule $r_{4}$ confirms to the long-standing expectations of baseball fans

$c s_{1}$ : rule $x$ confirms to the long-standing expectations of baseball fans $\leadsto$ rule $r$ is adopted by the relevant practice of baseball 
Rule $r_{4}$ has an exception in case the ball is not loose when retrieved, for instance, when someone else achieved a right to the ball but involuntarily lost control. The effect of this exception is shown in Fig. 2, which displays why Hayashi cannot win by rules $r_{4}$ and $r_{1}$. (Alternatively, such cases could be modelled as giving rise to an argument that the ball was not abandoned property any more when Hayashi picked it up. In this interpretation of the concept 'abandoned property' the counterargument in Fig. 2 must be targeted at the premise 'The baseball was abandoned property'.)

$r_{5}$ : the ball is not loose when $x$ retrieves it $\leadsto$ rule $r_{4}$ is not applicable

$r_{6}$ : ( $x^{\prime}$ has possession of the baseball $\vee x^{\prime}$ has a right to possession of the baseball $\vee x^{\prime}$ has a qualified right to possession of the baseball) $\wedge x^{\prime}$ involuntarily lost control of the baseball $\wedge x^{\prime} \neq x \leadsto$ the ball is not loose when $x$ retrieves it

(The point of the second and third disjunct of the first condition will become apparent in Sect. 3.2.2.) The validity of $r_{5}$ and $r_{6}$ was not discussed and will therefore be stated as assumptions. Now while $r_{2}, r_{3}$ and $r_{4}$ were uncontroversial in the case, a closer analysis of the decision shows that $r_{3}$ in fact compresses a more detailed line of reasoning, as will be explained below after rules $G_{2 a}$ and $G_{2 b}$.

Since it is not in dispute that Hayashi retrieved the ball, Popov must provide an argument that the ball was not loose when Hayashi retrieved it, for instance, that he had gained possession but then lost control because of the illegal attack by the mob. Since it is not in dispute that he was illegally attacked by the mob and that he at least attempted to catch the ball in order to take possession, the crucial issue is the definition of possession.

At this point McCarthy takes his departure in Professors Bernhardt and Brown's general definition of possession (not restricted to baseball games):

$B B_{1 a}: \quad x$ has physical control over item $y \wedge x$ intends to control $y \leadsto$ $x$ has possession of $y$

$B B_{1 b}$ : $x$ has no physical control over item $y \vee x$ does not intend to control $y \leadsto x$ $x$ does not have possession of $y$

Assuming that professors Bernhardt and Brown are legal experts, these rules are valid by validity rule $v_{2}$ (likewise below for the further rules proposed by professors of law). Popov's intention to physically control the ball $\left(f_{8}\right)$ was not in dispute. The factual issue was whether he had obtained physical control by his 'snowcone' catch. In addition, there is the legal issue whether physical control is indeed a necessary condition for possession.

McCarthy next discusses two alternative ways to use Bernhardt and Brown's rule to "craft" a definition of possession that is specific to the context of baseball games. He first presents Professor Gray's opinion, who first adopts $r_{3}$ above and then states:

$G_{1 a}: \quad x$ has complete control of the baseball when momentum of $x$ and baseball cease $\leadsto x$ catches the baseball

$G_{1 b}: \quad x$ does not have complete control of the baseball when momentum of $x$ and baseball cease $\leadsto x$ does not catch the baseball 


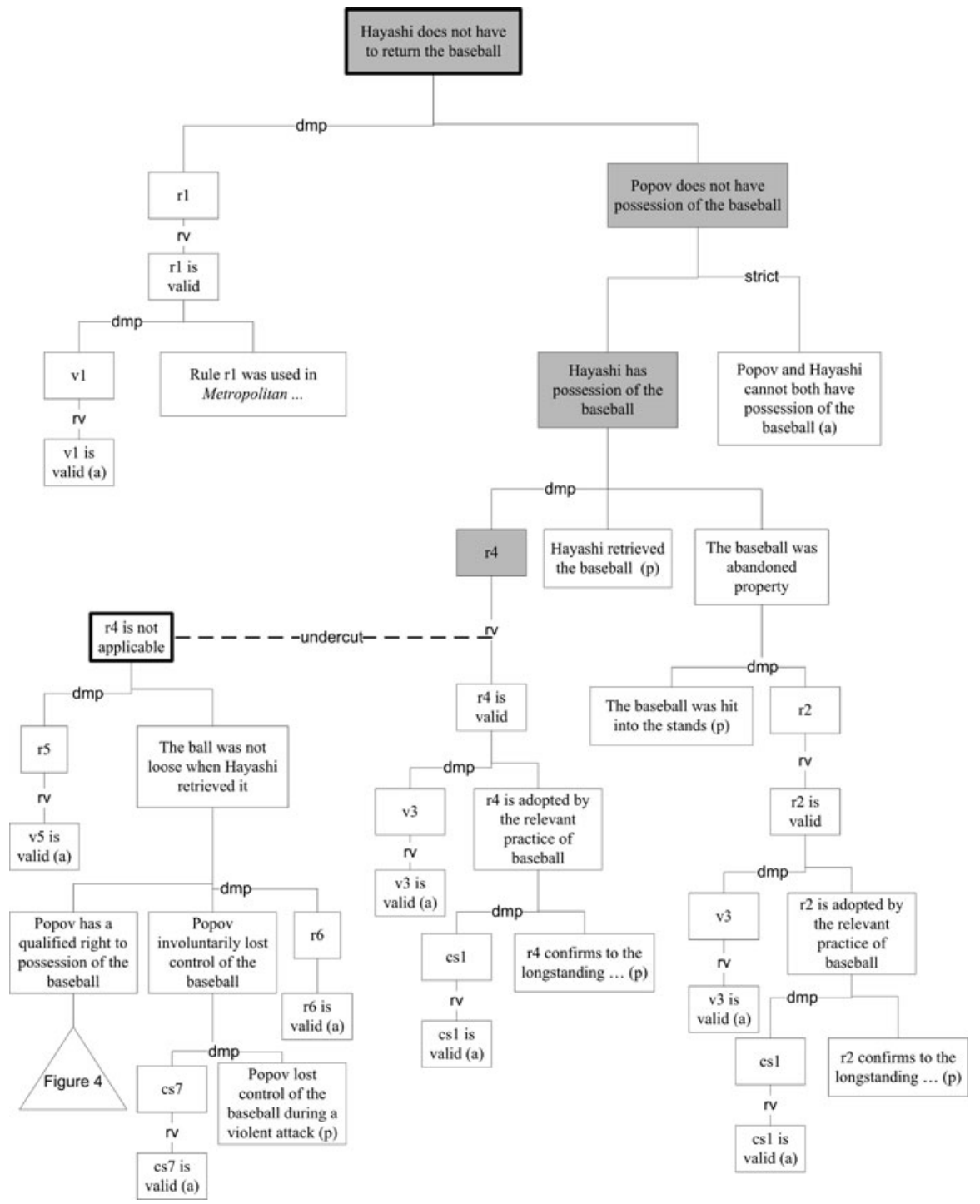

Fig. 2 An overruled argument why Hayashi does not have to return the baseball

How can we see these rules as a specialisation of Bernhardt and Brown's rule to a baseball context? An obvious way is to add the rules

$G_{2 a}: \quad x$ catches the baseball $\leadsto x$ has physical control over the baseball

$G_{2 b}: \quad x$ does not catch the baseball $\wedge x$ does nothing else to gain physical control $\leadsto x$ does not have physical control over the baseball

The specialisation thus takes the form of specifying a way to derive the condition of $B B_{1 a}$ or $B B_{1 b}$ in the context of a baseball game. Note that on this account $r_{3}$ turns out 
to be a compression of a line of reasoning, namely, by hypothetically assuming its conditions, then applying $G_{2 a}$ to them and then applying $B B_{1 a}$ to the thus derived consequent of $G_{2 a}$. Such compressions were analysed by Loui and Norman (1995) as a kind of case rationale and recently by Bench-Capon and Prakken (2010) as a way to generate tests in US Supreme Court oral arguments. Alternatively, $r_{3}$ can be retained in its original form: the result is then the same but the logical connection with $B B_{1 a}$ and $B B_{1 b}$ is lost.

Professor Gray then says that a baseball which is dislodged by incidental contact with an inanimate object or another person is not possessed. This can be regarded as specifying a case in which the first condition of $G_{1 a}$ is not satisfied. On this account, Professor Gray again compresses a hypothetical line of reasoning, this time using the following rule:

$G_{3}: \quad x$ lost the baseball before momentum of $x$ and baseball ceased $\wedge$ $x$ lost the baseball by incidental contact $\leadsto$

$x$ does not have complete control of the baseball when momentum of $x$ and the baseball cease

The hypothetical line of reasoning starts by assuming the conditions of $G_{3}$ and then applies $G_{3}$ to them to derive that $x$ does not have complete control of the baseball when momentum of $x$ and the baseball cease. This conclusion then satisfies the condition of $G_{1 b}$, which leads to the conclusion that $x$ does not catch the baseball. Hence $G_{2 a}$ cannot be applied so there is no way to apply $B B_{1 a}$ to conclude that Popov gained possession of the ball. It is this hypothetical line of reasoning that is arguably compressed in professor Gray's statement that a baseball which is dislodged by incidental contact with an inanimate object or another person is not possessed.

McCarthy next discusses professors Bernhardt and Finkelman's alternative definition of possession, according to which possession can also result if no complete physical control is achieved:

$B F_{1}: \quad x$ is actively and ably engaged in efforts to establish complete control of item $y \leadsto x$ possesses item $y$

$B F_{2}: \quad x$ intends to take control of a baseball $\wedge$

$x$ manifests this intention by stopping the forward motion of the ball $\leadsto x$ is actively and ably engaged in efforts to establish complete control of the baseball

These rules are valid since they are the rationale of a set of precedents concerning the fishing and hunting of wild animals and the salvage of sunken vessels (such as the famous triple Pierson v. Post, Keeble v. Hickeringill and Young v. Hitchens, widely discussed in AI and Law). In addition, McCarthy gives a further reason why the exception is valid, namely that this rule is influenced by the custom and practice in each of these industries.

Since McCarthy explicitly discusses which rule he should adopt in the present case, an analysis in terms of applicability of valid rules seems more suitable than an analysis in terms of priority between conflicting valid and applicable rules. More precisely, McCarthy says that $B F_{1}$ applies in contexts where it is impossible to achieve complete physical control of an item, as in the fishing and hunting of wild animals and the salvage 
of sunken vessels, while Gray's analysis applies in contexts where such control is possible. Since the undercutter for defeasible modus ponens make rules applicable by default, the following inapplicability rules suffice to capture this analysis:

$a_{1}$ : full physical control is impossible $\leadsto$ applying $B B_{1 b}$ is unworkable and unreasonable

$a_{2 a}$ : applying rule $r$ is unworkable and unreasonable $\leadsto$ rule $r$ is not applicable

$a_{2 b}$ : full physical control is possible $\leadsto$ rule $B F_{1}$ is not applicable

McCarthy leaves the validity of these rules implicit.

After thus having adopted professor Gray's analysis, McCarthy observes that Popov did not lose control of the ball by incidental contact but by an intended attack by a violent mob, so that Gray's rule $G_{3}$ cannot be used to argue that Popov did not achieve possession of the baseball: one condition of this rule is not fulfilled; not even an argument can be built for it. He then discusses whether Popov would have gained control over the ball had he not been attacked. Here he seems to read a counterfactual element into Gray's rules; this reading can be captured by modifying $G_{1 a}$ and $G_{1 b}$ as follows:

$G_{1 a}: \quad x$ has complete control of the baseball when momentum of $x$ and baseball cease $\vee(x$ lost control of the baseball during a violent attack $\wedge x$ would have had complete control of the baseball when momentum of $x$ and baseball ceased if $x$ had not been attacked) $\leadsto$

$x$ catches the baseball

$G_{1 b}$ : $x$ does not have complete control of the baseball when momentum of $x$ and baseball cease $V$

( $x$ lost control of the baseball during a violent attack $\wedge$

$x$ would not have had complete control of the baseball when momentum of $x$ and baseball ceased if $x$ had not been attacked) $\leadsto$

$x$ does not catch the baseball

McCarthy next applies the counterfactual element of this rule to the facts, observing that

Mr. Popov has not established by a preponderance of evidence that he would have retained control of the ball after all momentum ceased and after any incidental contact with people or objects. Consequently, he did not achieve full possession. (p. 8)

McCarthy here observes in passing that Popov has the burden of persuasion for his claim that he would have retained control. It therefore seems justified to interpret his remark that Popov did not achieve full possession as saying that Popov has not been able to prove full possession. This is a metalevel observation about what can be concluded from the available arguments. Now there was some evidence for Popov's claim, namely the facts $f_{1}$ (Popov stopped the forward motion of the baseball) and $f_{2}$ (The baseball was in the glove of Popov) and Popov's own testimony:

$e_{1}$ : Witness Popov says he had full control of the baseball when he was attacked 
From Popov's testimony and the following obvious commonsense rule

$c s_{2}: \quad x$ had full control of the baseball when he was attacked $\leadsto$ $x$ would have had complete control of the baseball when momentum of $x$ and baseball ceased if $x$ had not been attacked

an argument from witness testimony can be constructed, which is undercut by McCarthy with the following words and implicit commonsense rule:

$e_{2}$ : Mr. Popov's testimony on this question is inconsistent on several points, ambiguous on others and, on the whole, unconvincing

$c s_{3}: \quad e_{2} \leadsto$ Popov is not credible

Next, from $f_{1}$ and $f_{2}$ an argument can be constructed if the following commonsense generalisation is assumed to be valid:

$c s_{4}: \quad f_{1} \wedge f_{2} \leadsto$ Popov would have had complete control of the baseball when momentum of Popov and baseball ceased if Popov had not been attacked

Then McCarthy's statement that Popov's claim cannot be proven implies an argument against the validity of this rule, which can arguably be based on facts $f_{3}$ (Popov was in motion when he was attacked) and $f_{6}$ (Popov lost contact with the baseball during the violent attack).

$c s_{5}: f_{3} \wedge f_{6} \leadsto c s_{4}$ is not valid

Now that both of Popov's arguments that he would have retained full control of the baseball if he had not been attacked are defeated, their accrual is also defeated (see Fig. 3).

\subsection{2 (Qualified) right to possession}

After having decided that Popov never achieved full possession of the ball (Fig. 3), McCarty turns to the second disjunct of the first condition of $r_{1}$, namely, whether Popov at least achieved a right to possession. He observes that in cases like this one, where the court "sits in equity", it has the authority to state its own rules "to achieve fundamental fairness":

$v_{4}: \quad$ rule $r$ concerns legal action $x \wedge x$ is an action in equity $\wedge$

rule $r$ promotes fundamental fairness as regards $x \wedge$

court $c$ adopts rule $r$ to decide $x \leadsto$ rule $r$ is valid

$v_{4}$ : rule $r$ concerns legal action $x \wedge x$ is an action in equity $\wedge$ rule $r$ does not promote fundamental fairness as regards $x \leadsto$ rule $r$ is not valid

This validity rule, of which McCarthy leaves the validity implicit, seems a key element in the decision, since without it McCarthy may not have had any legal ground for the decision he considers fair.

McCarthy then proposes the following rule:

$M C_{1}: \quad x$ undertakes significant but incomplete steps to achieve possession of $y \wedge$ $y$ is abandoned property $\wedge$ 


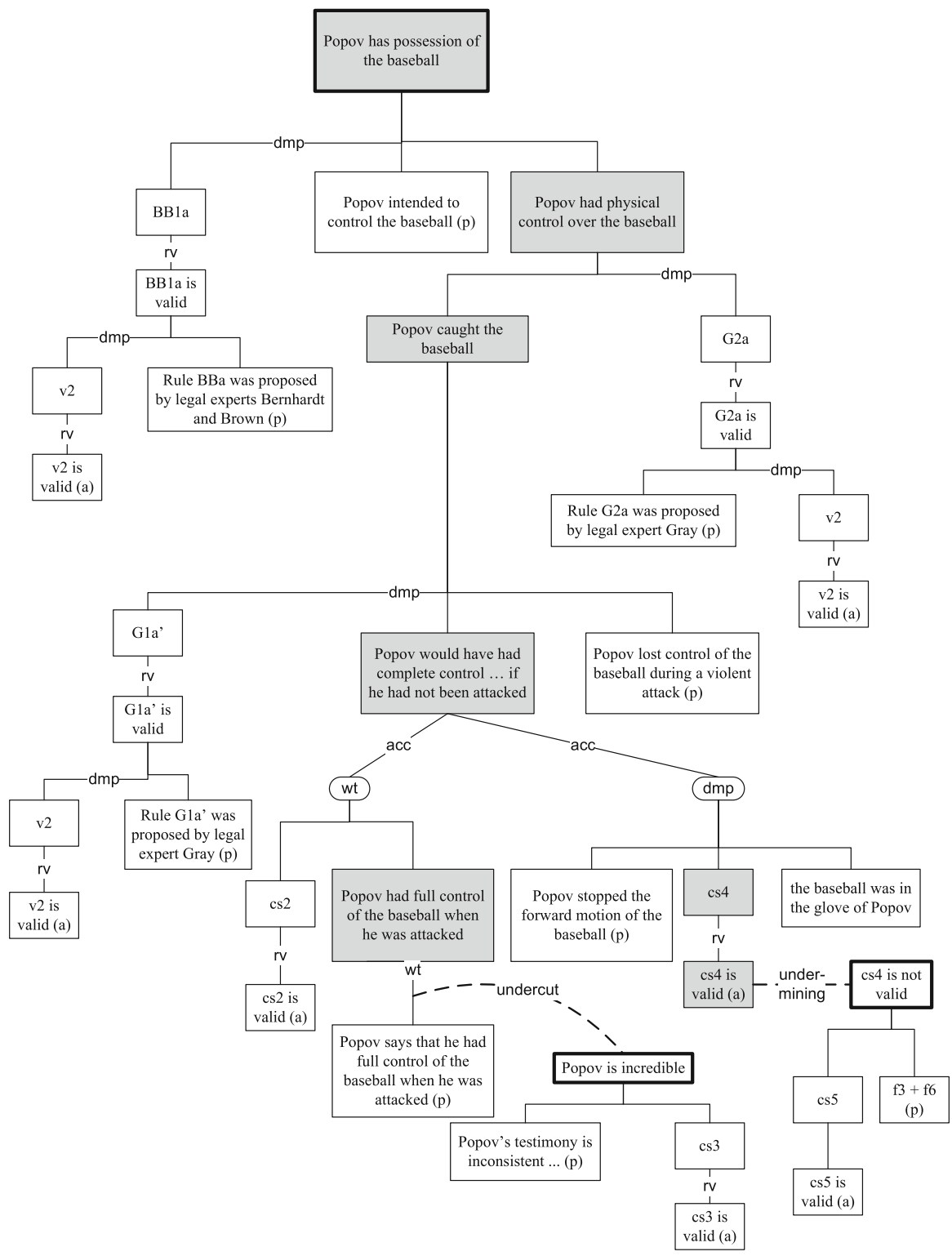

Fig. 3 An overruled argument why Popov has possession of the baseball

the effort of $x$ is interrupted by unlawful acts of others $\leadsto$ $x$ has a legally cognizable pre-possessory interest in $y$

and states that it is "consistent" with the principle formalised above as $v_{4}$. Thus he in fact states that all conditions of $v_{4}$ are satisfied for this rule. From McCarthy's statements it seems obvious that he also assumes the following rules: 
$M C_{2}: \quad x$ has a legally cognizable pre-possessory interest in $y \leadsto$ $x$ has a qualified right to possession of $y$

$M C_{3}$ : $\quad x$ has a qualified right to possession of $y \leadsto x$ has no right to possession of $y$ $c s_{6}$ : $\quad x$ has a qualified right to possession of $y \leadsto z$ has a legitimate claim to $y$

Figure 4 displays the argument why Popov has a legitimate claim to the baseball. Note the word "qualified" in the consequent of $M C_{2}$, which makes it insufficient to fulfill the second disjunct of the first condition of $r_{1}$, so that further rules are needed if Popov's interest is to be satisfied.

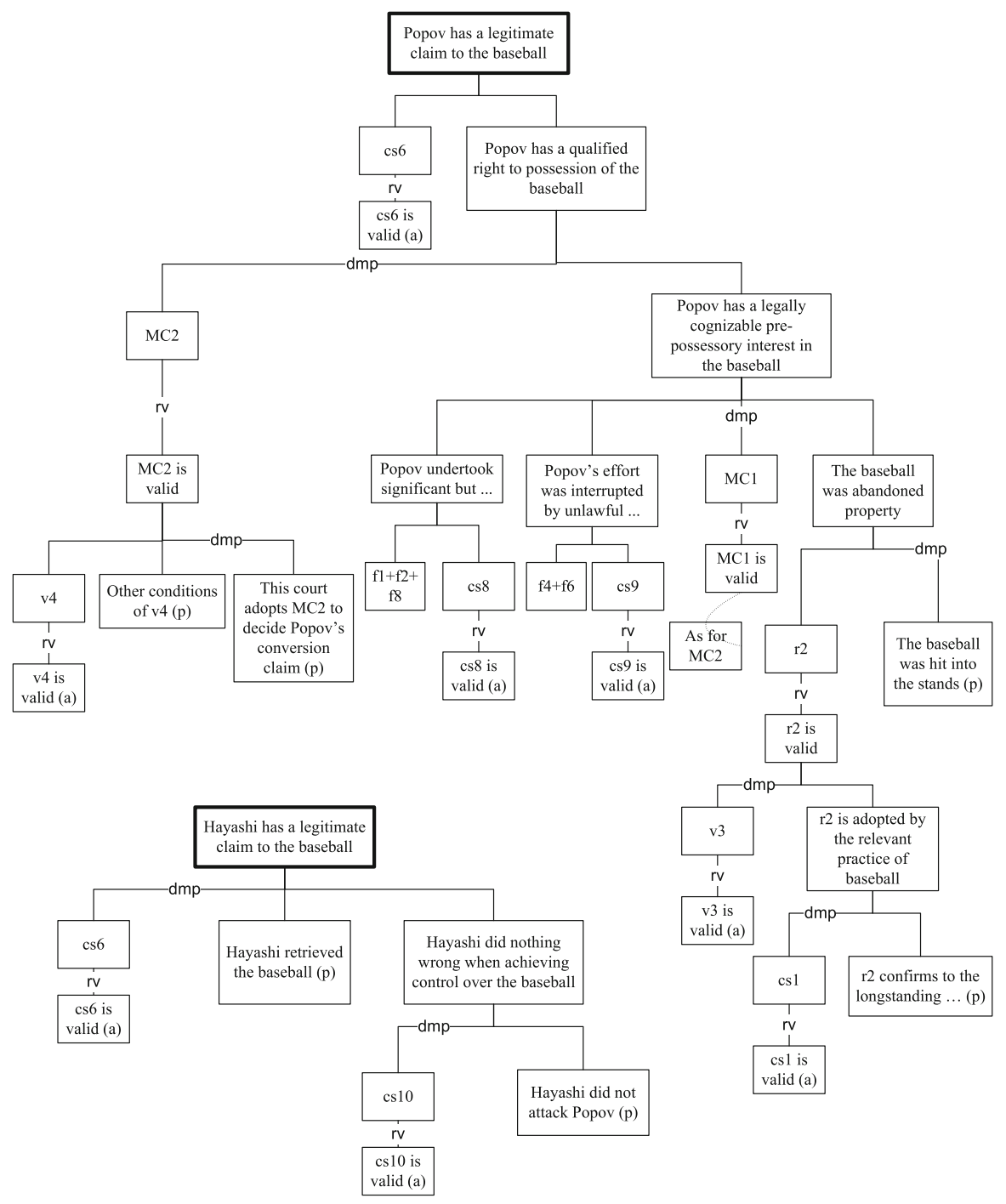

Fig. 4 Justified arguments that both Popov and Hayashi have a legitimate claim 
McCarthy now first says that "The court is required to balance the interests of all parties". This can be read as saying that a rule that does not balance the interests of all parties is unfair:

$M C_{4}$ : rule $r$ does not balance the interests of all parties $\leadsto$ rule $r$ does not promote fundamental fairness as regards $x$

McCarthy leaves the validity of this rule implicit. He then observes that Hayashi also has legitimate interests in the baseball (see also the second argument in Fig. 4).

$M C_{5 a}: \quad z$ retrieved $y \wedge z$ did nothing wrong when achieving control over $y \leadsto z$ has a legitimate claim to $y$

$M C_{5 b}: \quad x$ has possession of $y \vee x$ has the right to possession of $y \leadsto$ $M C_{5 \mathrm{a}}$ is not applicable

McCarthy then first rejects the following two candidate decision rules as not taking the interests of both parties into account (see the arguments in Fig. 5):

$r_{p}: \quad x$ has a qualified right to possession of $y \wedge z$ holds $y \wedge$

$x$ demands $z$ to return $y \leadsto z$ must return $y$ to $x$

$r_{h}$ : $z$ has a legitimate claim to $y \leadsto z$ does not have to return $y$ to $x$

Then McCarthy arguably states the following two rules (again leaving their validity implicit), which together and combined with $M C_{4}$ say that if both parties have a legitimate claim to a property, deciding for one of them is unfair:

$M C_{6}: \quad x$ has a legitimate claim to $y \wedge x$ is defendant in a conversion case $\wedge$ rule $r$ finds for plaintiff $\leadsto$

rule $r$ does not balance the interest of all parties

$M C_{7}: \quad x$ has a legitimate claim to $y \wedge x$ is plaintiff in a conversion case $\wedge$ rule $r$ finds for defendant $\leadsto$

rule $r$ does not balance the interest of all parties

It is interesting to see what happens if, in McCarthy's words, a psychic can tell whether Popov would have achieved full control had he not been attacked by the mob. If Popov would have had complete control of the baseball without the violent attack, then the conditions of $M C_{5 b}$ can be derived via $G_{1 a}$, so $M C_{5 a}$ cannot be used to derive that Hayashi has a legitimate interest in the ball. So in that case Popov wins by $r_{3}$. If, on the other hand, Popov would not have had complete control of the baseball without the violent attack, then arguably a commonsense rule applies that his steps to achieve possession of the baseball were not significant so that the first condition of $M C_{1}$ is not satisfied and it cannot be derived that Popov has a legitimate interest in the baseball. Moreover, in that case $G_{1 b}$, makes that Popov did not achieve possession, so in that case Hayashi wins by $r_{4}$. Thus the present reconstruction captures that McCarthy's decision crucially depends on the fact that it cannot be proven what would have happened had Popov not been attacked.

Finally, after rejecting the rules that find for either Popov or Hayashi, McCarthy justifies his decision to equally divide the profit over both (his deciding argument is visualised in Fig. 6). Here his arguments must be interpreted with care. On the one hand, McCarthy has just observed that he has the authority to formulate rules that 


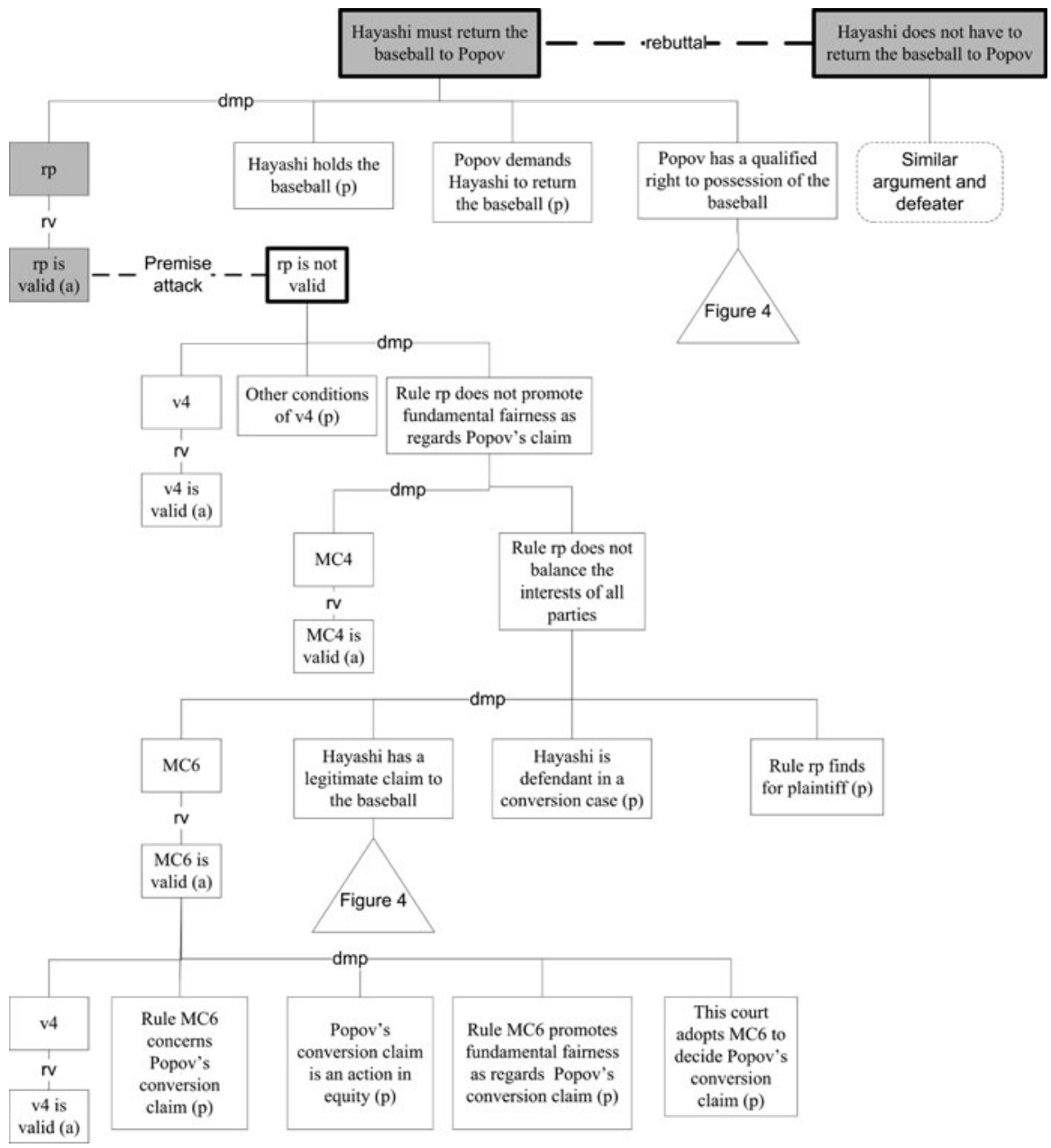

Fig. 5 Overruled arguments that Popov or Hayashi should win

promotes fairness. On the other hand, he now justifies his choice for the deciding rule by referring to one legal scholar who proposed it and two precedents in which it was used. My interpretation is that McCarthy only uses $v_{4}$ to fill the gap between the facts and the rules that he can 'find' in the law. On this account, the rule found by him is the one suggested by legal expert Helmholz and the two precedents, while the other rules $\left(M C_{1}-M C_{7}\right)$ are 'fashioned' by McCarthy and then combined with Helmholz' rule to establish that Popov and Hayashi have equal rights to the baseball (although $M C_{4}$ instead seems a legal commonsense rule on what it means to promote fundamental fairness).

This interpretation can be formalised as follows. To start with, it should be noted that the "fashioning" of rules to achieve fundamental fairness in the case is not a problem of finding the valid and applicable legal rule but a problem of choice, that 


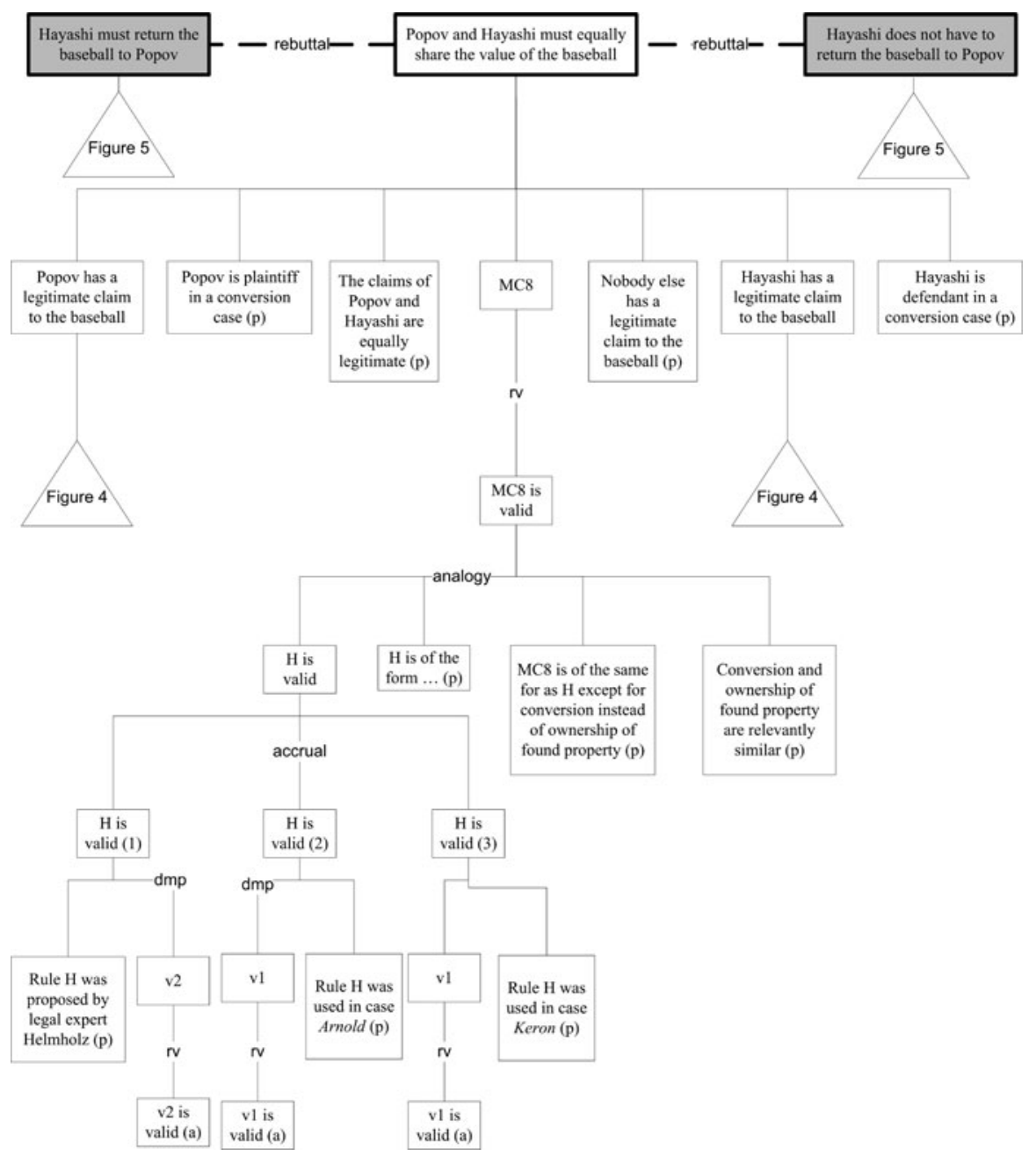

Fig. 6 The deciding argument

is, a problem of practical reasoning: faced with the goal to adopt a rule that promotes fundamental fairness in the case, McCarthy must ideally consider all available ways to achieve that goal, that is, all rules that achieve fairness in this case, and then make a rational choice given his values and preferences. Ideally, this reasoning process would be captured by applications of practical reasoning argument schemes like those proposed by Atkinson and Bench-Capon (2007) and Bench-Capon and Prakken (2006b). However, since McCarthy does not consider any alternative rules that promote fundamental fairness, his decision will simply be stated as a fact. It may be that McCarthy's internal deliberations were much more involved and contained rejections of alternative ways to promote fundamental fairness in the case, but we cannot know this from the text. 
The rule that in the present interpretation is 'found' by McCarthy is

$M C_{8}: \quad x$ has a legitimate claim to property $y \wedge x$ is plaintiff in a conversion case $\wedge$ $z$ has a legitimate claim to property $y \wedge z$ is defendant in a conversion case $\wedge$ the claims of $x$ and $z$ are equally legitimate $\wedge$ nobody else has a legitimate claim to $y \leadsto$ $x$ and $z$ must equally share the value of property $y$

It seems that McCarthy derives the validity of this rule by three accruing validity arguments, grounding the rule, respectively, in legal expert Helmholz's writings and in two precedents. To formalise this, the following three relevant 'legal' facts must be stated and combined with, respectively, validity rules $v_{2}$ and $v_{1}$.

$l_{5}$ : rule $H$ was proposed by legal expert Helmholz

$l_{6}$ : $\quad$ rule $H$ was was used in case Arnold v. Producers Fuit Company

$l_{7}$ : rule $H$ was was used in case Keron $v$. Cashman

(Here it is for simplicity ignored that Arnold v. Producers Fuit Company in fact used a slightly more general rule, referring to the proportionality of the competing legal claims.) The reason that these legal facts use the rule name $H$ instead of $M C_{8}$ is that there is a complication here: expert and precedents proposed and used the rule for a different type of case than conversion, namely, to decide competing claims between finders of lost or mislaid property and the owners of the land on which the property was found. McCarthy thus arguably uses the following argument scheme for analogical reasoning with legal rules:

Analogical rule validity scheme:

$r$ is valid,

$r$ is of the form

$r: \quad x$ has a legitimate claim to property $y \wedge$

$x$ is plaintiff in a case of type $c \wedge$

$z$ has a legitimate claim to property $y \wedge$

$z$ is defendant in a case of type $c \wedge$

the claims of $x$ and $z$ are equally legitimate $\wedge$

nobody else has a legitimate claim to $y \leadsto$

$x$ and $z$ must equally share the value of property $y$

$r^{\prime}$ is of the same form but with $c$ replaced with $c^{\prime}$,

$c$ and $c^{\prime}$ are relevantly similar

$\Rightarrow$

$r^{\prime}$ is valid

More general versions of this scheme might be used in which the other conditions and consequents of the rules are similar instead of equal, but this is not supported by McCarthy's statements. An obvious question is why the cases of conversion and finding of property are similar. If McCarthy had made his reasoning at this point explicit, then sophisticated AI (\& Law) models of analogical reasoning might have been needed, but this is beyond the text of his decision and therefore beyond the 
scope of this paper. The main point of the present reconstruction is to show that at least some forms of legal analogical reasoning can be formalised in the framework of Prakken (2010) as defeasible inference rules.

\section{The arguments and their evaluation}

\subsection{The arguments}

The most important arguments resulting from the above analysis are displayed in Figs. 2, 3, 4, 5, 6. As for notation, the type of premise is indicated with (p) for ordinary premises and (a) for assumptions. Some but not all of these facts were listed above in Sect. 3.1. Of those that were not listed above the instantiation of the variables with constants should be obvious. The inference links are labelled with the name of the argument scheme that is applied. In the figures that contain counterarguments, the defeated parts of arguments, that is, the defeated subarguments, are shown in grey colour.

Some specific comments:

- In Fig. 2 the statement that Popov and Hayashi cannot both have possession of the baseball is formalised as an assumption premise since, although it is presumably based on general property law, it cannot be traced back to explicit statements in the decision and instead seems to be implicitly assumed. Furthermore, the commonsense rule $c_{7}$ has the following form:

$c s_{7}$ : $x$ loses control of item $y$ during a violent attack $\leadsto$

$x$ involuntarily loses control of $y$

- In Fig. 3 the application of the accrual argument scheme is for reasons of space displayed in a compressed way; Fig. 6 shows its application in another argument in more detail.

- In Figs. 4 in the premises of ' $M C_{2}$ ' is valid and in Fig. 5 in the premises of " $r_{p}$ is not valid', some conditions of rule $v_{4}$ are summarised into one node; in the latter figure the application of $v_{4}$ is shown in full detail in the premises of ' $M C_{6}$ is valid'.

In Fig. 4 the commonsense rules $c s_{8}, c s_{9}$ and $c s_{10}$ have the following form:

css: $f_{1} \wedge f_{2} \wedge f_{8} \leadsto$ Popov undertook significant but incomplete steps to achieve possession of the baseball

$c s_{9}: \quad f_{4} \wedge f_{6} \leadsto$ Popov's efforts were interrupted by unlawful acts of others

$c s_{10}: f_{5} \wedge f_{6} \leadsto$ Hayashi did nothing wrong when achieving control of the baseball

- In Fig. 5 the proposition ' $r_{p}$ is valid' is formalised as an assumption since it was presumably suggested by Popov. Judge McCarthy's rejection of this rule thus becomes a defeater by way of premise attack. Alternatively, McCarthy's arguments could be regarded as a simple explanation that one premise to build the argument for 'Hayashi must return the baseball to Popov' is as a matter of fact false so that this argument cannot be constructed. 
- In Fig. 5 the argument that Hayashi does not have to return the baseball and its refutation are similar to the argument for 'Hayashi must return the baseball to Popov' and are therefore not visualised.

- In Figs. 5 and 6 the rebuttal relations are based on the choice to make the three top-level propositions contraries of each other in $\mathcal{L}$.

- To keep Figs. 5 and 6 readable, they do not display that the argument for 'Hayashi does not have to return the baseball' from Fig. 2 also rebuts and is rebutted by the arguments for the conclusions 'Hayashi must return the baseball' and 'Popov and Hayashi must equally share the value of the baseball'. For the same reason the figures do not visualise the accrual of the arguments for the conclusion 'Hayashi does not have to return the baseball' from Figs. 2 and 5. Note also that similar remarks apply to the extension of the argument in Fig. 3 to an argument for 'Hayashi must return the baseball'.

\subsection{Evaluating the arguments}

As remarked above, the framework of Prakken (2010) defines a set of arguments with a binary defeat relation (called an argumentation theory), so that the acceptability of arguments can be defined in terms of Dung's (1995) abstract semantics for argument acceptability (Dung called an argumentation theory an "argumentation framework"). Without going into details, all of Dung's semantics can be regarded as classifying arguments into three acceptability classes: the justified arguments (those that directly or indirectly survive all attacks), the overruled arguments (those that are defeated by a justified argument) and the defensible arguments (involved in conflicts that cannot be resolved). The main feature of Dung's semantics that is relevant for the present analysis is that they all capture the phenomenon of reinstatement: an argument with defeaters is still justified if all its defeaters are in turn defeated by a justified argument.

At first sight, it might be thought that the argumentation theory consists of the eleven arguments and their defeat relations shown in Figs. 2, 3, 4, 5, 6, that is, the elevent trees shown in these figures. However, this is not the case, since of each argument also all its subarguments are part of the theory. In graphical terms, all subtrees of the trees shown in the figures are also in the argumentation theory. Moreover, the theory also contains more defeat relations than displayed, since when an argument defeats another argument, it also defeats all its continuations with further inferences. So, for instance, in Fig. 2 the argument with conclusion 'Popov is incredible' not only defeats the argument with conclusion 'Popov had full control of the baseball when he was attacked' but also, for instance, the arguments with conclusion 'Popov would have had complete control ... if he had not been attacked' and 'Popov had possession of the baseball'.

Having said this, determining the acceptability status of McCarthy's deciding argument is still straightforward, whichever of Dung's (1995) semantics is used. As shown in Fig. 6, this argument is rebutted on its final conclusion by the two arguments that Popov, respectively, Hayashi should win. Judge McCarthy did not resolve these conflicts with preferences, so that both rebuttals result in symmetric 
defeat relations. Instead, McCarthy defeated the two rebuttals by premise-attacking them on their assumptions that rule $r_{p}$, respectively, rule $r_{h}$, are valid (the argument for ' $r_{p}$ is not valid' shown in Fig. 5 and the similar argument for ' $r_{h}$ is not valid' that is left implicit in this figure). Since an attack on an assumption always results in asymmetric premise-defeat and since the two defeaters themselves have no defeaters, they are both justified.

However, this is not enough to reinstate McCarthy's deciding argument, since it has two further defeaters. Firstly, as remarked above, the argument in Fig. 3 can be extended to another argument for the conclusion that Hayashi must return the baseball, based on the subargument for the conclusion that Popov has possession of the baseball. This argument also rebuts (and is rebutted by) the arguments for the conclusions that Hayashi does not have to return the baseball and that Popov and Hayashi must equally share its value. This rebuttal is overruled in a similar way as just explained, by an argument that strictly defeats one of its subarguments and thereby reinstates McCarthy's deciding argument. More specifically, this holds for the subargument for 'Popov had full control of the baseball when he was attacked'. In addition, the argument in Fig. 3 is also defeated by an attack on its assumption premise that $c s_{4}$ is valid. A similar analysis applies to the argument in Fig. 2 for the conclusion that Hayashi does not have to return the baseball to Popov. This argument rebuts (and is rebutted by) the arguments for the conclusions that Hayashi must return the baseball and that Popov and Hayashi must equally share its value. However, it is still overruled since its subargument for the conclusion that Hayashi has possession of the baseball (in turn based on the fact that he retrieved it) is undercut since the fact that Popov has a qualified right to possession of the baseball is an exception to rule $r_{4}$.

A final complication is that, as also remarked above, the two arguments for the conclusion that Hayashi must return the baseball accrue since they are both defeasible, and that the same holds for the two arguments that Hayashi does not have to return the baseball. However, this does not change the above outcome, since for both accrual arguments their accruing subarguments are defeated by justified arguments, so that the same holds for the accruals.

Finally, how does this analysis account for the burden of proof? In the present case it was uncontroversial that Popov had the burden of persuasion for his main claim, with as proof standard 'preponderance of evidence' and that there were no shifts in the burden of persuasion for subissues. As suggested by Prakken and Sartor (2009) this can be modelled in the present framework by requiring that Popov wins if and only if a justified argument for his main claim can be constructed. Proof standards are in Prakken and Sartor's (2009) approach applied in two ways. The first is when deciding whether one of two rebutting arguments strictly defeats the other, that is, in defining the preference relations with which the conflict can be resolved: the lower the proof standard, the easier the preference will be defined such that one argument strictly defeats the other. In the present case this way of applying proof standards did not occur. The second way to apply a proof standard is in deciding whether an undercutter or invalidity argument must be moved against an evidential argument: the lower the proof standard, the easier such a counterargument will be moved. In the present reconstruction this way of applying proof standards occurred in deciding whether to formulate the rules $c s_{3}$ and $c s_{5}$. 


\section{Discussion}

The aim of this paper was to test the suitability of the abstract framework of Prakken (2010) for modelling the argumentation structure of judge McCarthey's decision. Although a definite conclusion can only be drawn after comparing the present reconstruction with the other ones submitted to this issue, it can be tentatively concluded that the formalism is suitable. The abstract rule-based nature of the formalism turned out to be suitable for modelling the argument schemes used in the case, while the tree-structure of the arguments captured the dependencies between the elements in the judge's decision. Also, the three classes of conflict between arguments were sufficient to capture all conflicts in the case and Dung's (1995) acceptability semantics sufficed for capturing the outcome of the case, including a proper handling of the burden of proof. The two main technical devices that were needed were rule-exception structures and arguments about the validity of rules. The latter proved sufficient to capture value-based reasoning, since the only value that was at stake in this case, fairness, was reconstructed as a condition of a validity rule. For this reason more sophisticated models of value-based reasoning, such as those of Prakken (2002) or Bench-Capon (2003), in which values are used to resolve conflicts between arguments, were not needed. Arguments about validity also proved useful in modelling the use of cases, since cases were by McCarthy mostly used as sources of rules (see also Verheij 2008). There was no need to use more sophisticated models of legal case-based reasoning such as those discussed by Ashley (2006) but there was a need for an argument scheme for analogical rule application. Finally, the present reconstruction contains some examples of argument accruals but they were not relevant for the outcome of the case.

It is interesting to compare the present reconstruction with the only reconstruction available in the literature, the one of Wyner et al. (2007). Being a conference paper, their reconstruction is less detailed than the present one. Wyner et al. also make use of domain-specific defeasible rules and argument schemes; like the present paper they use defeasible modus ponens and schemes for witness testimony and video tape, while in addition they use a 'scheme from purpose' to model the teleological aspects of the case. The legal analysis is mostly reconstructed with ruleexception structures; no rule validity rules are used. One main difference with the present reconstruction is in the treatment of socio-legal values. While in the present reconstruction the inclusion of the fairness condition in the validity rule $v_{4}$ made McCarthy's considerations on fairness a matter of rule validity, in Wyner et al.'s reconstruction values are used to resolve conflicts between arguments (based on Bench-Capon 2003). In my opinion, the present account is closer to the actual wording of McCarthy's decision. In fact, besides fairness Wyner et al. include a second value of public order in their analysis. In the present reconstruction this value was not included since it cannot easily be based on statements of McCarthy.

A second main difference is that Wyner et al. use a different conception of arguments, in which each applicable rule gives rise to a separate argument in an abstract argumentation framework in the sense of Dung (1995) and in which there are no subargument relations between arguments. Instead, an argument applying a rule 'if $q$ then $p$ ' can be attacked by an argument 'not $p$ ?', which can then be 
counterattacked with an argument applying a rule 'if $r$ then $q$ '. Applying any of Dung's (1995) then yields that the argument for $p$ is reinstated by the argument for $q$, so that the arguments for $p$ and $q$ are justified and the argument 'not $q$ ?' is overruled. In the present approach instead a two-steps argument is created with premise $r$, intermediate conclusion $q$ and final conclusion $p$, and no counterargument is needed. In my opinion, this better captures the dependency relations between the elements of arguments, since complex arguments are usually not expressed with the attack-counterattack relations of 2007's method. It seems better to regard 'not $q$ '? not as stating a counterargument but as a separate kind of speech act, namely a challenge 'why $q$ ?' of the premises of an argument, which can be replied to with a further argument for that premise. Such speech acts can be modelled by embedding an argumentation logic in a dialogue system for argumentation, as in e.g. Gordon (1994); Prakken (2005c). For a case study that applies this idea see Prakken (2008).

An important question is to what extent the results of the present case study can be generalised. Strictly speaking this requires an experiment with a substantial number of cases but this goes beyond the scope of the present issue. A comparison can be made, however, with Prakken (2008), in which a similar formalism was applied to a reconstruction of a Dutch civil ownerships dispute. In that case study not just the final decision was reconstructed but also the preceding stages of the proceeding, for which reason in Prakken (2008) I had to embed a model of argument-based inference in a dialogue model of legal argument. As for the inferential part, the earlier case study also used rule-exception structures and the argument scheme from witness testimony, while in addition it used priority arguments; on the other hand, no validity arguments or arguments about values were used. The case contained an explicit shift in the burden of persuasion, which required the use of Prakken's (2001) adapted definition of justification of arguments.

As I observed earlier in Prakken (2008), case files often leave a considerable part of the relevant law implicit, since the law is assumed known to all parties. In Prakken (2008) this was not a serious problem, since the case was from a jurisdiction with which I am familiar. However, in the present case this is different. In experiments like the present one, where researchers are invited to perform case studies in jurisdictions with which they may not be familiar, this is an inevitable methodological problem. Nevertheless, when interpreted with care, case studies are a necessary complement to designs of formalisms or architectures, to reduce the risk that these designs are based on too simple accounts of legal reasoning.

Open Access This article is distributed under the terms of the Creative Commons Attribution License which permits any use, distribution, and reproduction in any medium, provided the original author(s) and the source are credited.

\section{References}

Amgoud L, Bodenstaff L, Caminada M, McBurney P, Parsons S, Prakken H, van Veenen J, Vreeswijk G (2006) Final review and report on formal argumentation system, Deliverable D2.6, ASPIC IST-FP6002307

Ashley K (2006) Case-based reasoning. In: Lodder A, Oskamp A (eds) Information technology and lawyers: advanced technology in the legal domain, from challenges to daily routine, Springer, Berlin, pp 23-60 
Atkinson K, Bench-Capon T (2007) Practical reasoning as presumptive argumentation using action based alternating transition systems. Artif Intell 171:855-874

Bench-Capon T (2003) Persuasion in practical argument using value-based argumentation frameworks. J Logic Comput 13:429-448

Bench-Capon T, Prakken H (2006a) Argumentation. In: Lodder A, Oskamp A (eds) Information technology and lawyers: advanced technology in the legal domain, from challenges to daily routine, Springer, Berlin, pp 61-80

Bench-Capon T, Prakken H (2006b) Justifying actions by accruing arguments. In: Dunne P, Bench-Capon T (eds) Computational models of argument. Proceedings of COMMA 2006, IOS Press, Amsterdam etc, pp 247-258

Bench-Capon T, Prakken H (2010) Using argument schemes for hypothetical reasoning in law. Artif Intell Law 18:153-174

Bex F (2009) Evidence for a good story. A hybrid theory of arguments, stories and criminal evidence, doctoral dissertation Faculty of Law, University of Groningen

Bex F, Prakken H, Reed C, Walton D (2003) Towards a formal account of reasoning about evidence: argumentation schemes and generalisations. Artif Intell Law 12:125-165

Dung P (1995) On the acceptability of arguments and its fundamental role in nonmonotonic reasoning, logic programming, and $n$-person games. Artif Intell 77:321-357

Gordon T (1994) The Pleadings Game: an exercise in computational dialectics. Artif Intell Law 2:239-292

Gordon T, Prakken H, Walton D (2007) The Carneades model of argument and burden of proof. Artif Intell 171:875-896

Hage J (1996) A theory of legal reasoning and a logic to match. Artif Intell Law 4:199-273

Loui R, Norman J (1995) Rationales and argument moves. Artif Intell Law 3:159-189

Pollock J (1994) Justification and defeat. Artif Intell 67:377-408

Prakken H (2001) Modelling defeasibility in law: logic or procedure?. Fundam Inform 48:253-271

Prakken H (2002) An exercise in formalising teleological case-based reasoning. Artif Intell Law 10:113-133

Prakken H (2005a) AI and law, logic and argument schemes. Argumentation 19:303-320

Prakken H (2005b) A study of accrual of arguments, with applications to evidential reasoning. In: Proceedings of the 10th international conference on artificial intelligence and law. ACM Press, New York, pp 85-94

Prakken $\mathrm{H}$ (2005c) Coherence and flexibility in dialogue games for argumentation. J Log Comput 15:1009-1040

Prakken H (2008) Formalising ordinary legal disputes: a case study. Artif Intell Law 16:333-359

Prakken H (2010) An abstract framework for argumentation with structured arguments. Argument Comput 1:93-124

Prakken H, Sartor G (1997) Argument-based extended logic programming with defeasible priorities. J Appl Non-Class Log 7:25-75

Prakken H, Sartor G (2009) A logical analysis of burdens of proof. In: Kaptein H, Prakken H, Verheij B (eds) Legal evidence and proof: statistics, stories, logic, Ashgate Publishing, Farnham, pp 223-253

Reed C, Rowe G (2004) Araucaria: Software for argument analysis, diagramming and representation. Int J AI Tools 13:961-980

Sartor G (2008) Legal policies and theories of legality: from ‘banana's' to Radbruch's formula. European University Institute Florence, EUI Working Papers Law 27

van den Braak S (2010) Sensemaking software for crime analysis, doctoral dissertation Department of Information and Computing Sciences, Utrecht University

Verheij B (2003) Dialectical argumentation with argumentation schemes: an approach to legal logic. Artif Intell Law 11:167-195

Verheij B (2008) About the logical relations between cases and rules. In: Francesconi E, Sartor G, Tiscornia D (eds) Legal knowledge and information systems. JURIX 2008: the twentyfirst annual conference, IOS Press, Amsterdam etc, pp 21-32

Vreeswijk G (1997) Abstract argumentation systems. Artif Intell 90:225-279

Walton D, Reed C, Macagno F (2008) Argumentation schemes. Cambridge University Press, Cambridge Wyner A, Bench-Capon T, Atkinson K (2007) Arguments, values and baseballs: representation of Popov v. Hayashi. In: Lodder A, Mommers L (eds) Legal knowledge and information systems. JURIX 2007: the twentieth annual conference, IOS Press, Amsterdam etc, pp 151-160 